\title{
Signals, Evolution and the Explanatory Power of Transient Information
}

\author{
Brian Skyrms \\ University of California, Irvine \\ bskyrms@uci.edu
}

\begin{abstract}
Pre-play signals which cost nothing are sometimes thought to be of no significance in interactions which are not games of pure common interest. We investigate the effect of pre-play signals in an evolutionary setting for Assurance, or Stag Hunt, games and for a Bargaining game. The evolutionary game with signals is found to have dramatically different dynamics from the same game without signals. Signals change stability properties of equilibria in the base game, create new polymorphic equilibria, and change the basins of attraction of equilibria in the base game. Signals carry information at equilibrium in the case of the new polymorphic equilibria, but transient information is the basis for large changes in the magnitude of basins of attraction of equilibria in the base game.
\end{abstract}

\section{Introduction}

Can signals that cost nothing to send and have any impact on strategic interaction? Folk wisdom exhibits a certain skepticism: "Talk is cheap." "Put you money where your mouth is." Diplomats are ready to discount signals with no real impact on payoffs. Evolutionary biologists emphasize signals that are too costly to fake. ${ }^{1}$ Game theorists know that costless signals open up the possibility of "babbling equilibria", where senders send signals uncorrelated with their types and receivers ignore the signals. Can costless signals have any explanatory power at all?

They can in certain kinds of benign interaction where it is in the common interest for signaling to succeed. In fact, even with potential signals that initially have no meaning, it is possible for signals to spontaneously acquire meaning under standard evolutionary dynamics and for such meaningful signaling to constitute an evolutionarily stable strategy. Such spontaneous emergence of signaling systems is always to be expected in strategic interactions such as the sender-receiver games introduced by David Lewis $(1969)^{2}$ to give a game-theoretic account of meaning. Signals acquire the ability to transmit information as a result of the evolutionary process. That this is so must be counted as one of the great successes of evolutionary game theory.

But what about interactions where the interests of the parties involved are not so perfectly aligned? In a bargaining game, one strategy may have no interest in whether signaling succeeds or not. In an assurance game one strategy may have an interest in communicating misinformation. How will the evolutionary dynamics of such games be affected if we add a round of costless pre-play signaling, allowing each player to condition her act on the signal received from the other player? It would not be implausible to expect that in such situations costless signals would have little of no effect. Talk is cheap. 
In fact, "cheap talk" signaling has a dramatic effect on the evolutionary dynamics of such interactions. That this is so should warn us against quick arguments against the effectiveness of costless signaling. Why it is so illustrates some subtleties of the role of information in the evolutionary process.

Section II briefly reviews the evolutionary dynamics in Sender-Receiver Games. Section III introduces the embedding of a two-person game in a larger "cheap-talk" game with a round of pre-play costless signaling by both players. Section IV discusses the effect of cheap talk on the evolutionary dynamics of an assurance game, where rational choice theory predicts that it should have no effect. Section V discusses the effect of cheap talk on the evolutionary dynamics of a bargaining game. Section VII concludes.

\section{Evolution of Meaning in Sender-Receiver Games}

In Convention, David Lewis introduced Sender-Receiver games to illustrate a game-theoretic account of conventions of meaning. One player, the sender, has private information about the true state of the world. The other player, the receiver, must choose an act whose payoff depends on the state of the world. The sender has signals available that she can send to the receiver, but they have no exogenously specified meaning. In the model of Lewis there are exactly the same number of states, signals and acts. In each state of the world there is a unique act that gives both players a payoff of 1 , all other acts giving a payoff of 0 .

A sender's strategy maps states of the world onto signals. A receiver's strategy maps signals onto acts. There are many Nash equilibria in this game, including ones in which the sender ignores the state of the world and always sends the same signal and the receiver ignores the signal and always chooses the same act. An equilibrium where players always get things right and achieve a payoff of 1 is called a Signaling System Equilibrium by Lewis. There are multiple signaling system equilibria. This makes Lewis' point that the meaning of the signals, if they have meaning, is conventional - it depends which signaling system equilibrium the players exemplify.

From the point of evolutionary game theory there are two striking facts about Lewis signaling games. The first has to do with the evolutionarily stable strategies. Recall that a strategy, s, is evolutionarily stable if, for any alternative strategy, m, s does better played against itself than $m$ does, or if they do equally well against $\mathrm{s}$, then $\mathrm{s}$ does better against $\mathrm{m}$ than $\mathrm{m}$ does. (If the latter condition is weakened from "s does better against $\mathrm{m}$ than $\mathrm{m}$ does" to "s does at least as well against $\mathrm{m}$ as $\mathrm{m}$ does" the strategy is said to be neutrally stable.)

In the evolutionary model for Lewis game, a player may find herself either in the role of sender or receiver. Her strategy is a pair <sender's strategy, receiver's strategy> from the original Lewis game. Although Lewis sender-receiver games have many 
equilibria other than signaling system equilibria, the signaling system equilibria coincide with the evolutionarily stable strategies in this evolutionary model. ${ }^{3}$

The second fact is more powerful. In a standard model of evolutionary dynamics, the replicator dynamics, the signaling system equilibria are attractors whose joint basin of attraction cover almost all of the possible population proportions. This is shown in simulations, where some signaling system or other always goes to fixation. In simplified sender-receiver games, it can be shown analytically. ${ }^{4}$ The analytical proof generalizes from the replicator dynamic to a broad class of adaptive dynamics, giving a demonstration of some robustness on the result.

\section{Evolutionary Games with Cheap Talk}

Given the effectiveness of adaptive dynamics in investing signals with meaning, it might be interesting to examine the coevolution of strategy in a game and of meaning in preplay signals preceding that game. In particular, consider a two-person symmetric game. We imbed this in a cheap talk game by introducing a set of signals, let each play send a signal to the other, and then let them play the base game where the act in the base game can depend on the signal received from the other player.

If there are $n$ possible signals then a strategy in the cheap talk game is an $n+1$ tuple:

$<$ signal to send, act to take if receive signal $1, \ldots$, act to take if receive signal $n>$. Thus a 2 by 2 base game with 2 signals generates a cheap talk game with 8 strategies; a 3 by 3 base game with 3 signals generates a cheap talk game with 81 strategies. If two strategies are paired in the cheap talk game, they determine acts in the base game and they receive the payoffs of their respective acts when those are paired in the base game.

Robson (1990) was the first to point out that cheap talk may have an important effect in evolutionary games. He considered a population of individuals defecting in the Prisoner's Dilemma. If there is a signal not used by this population, a mutant could invade by using this signal as a "secret handshake". Mutants would defect against the natives and cooperate with each other. They would then do better than natives and would be able to invade. Without cheap talk, a population of defectors in Prisoner's Dilemma would be evolutionarily stable. With cheap talk this is no longer true.

This is not to say that cheap talk establishes cooperation in the Prisoner's Dilemma. Mutants who fake the secret handshake and then defect can invade a population of the previous kid of mutants. And then if there is still an unused message, it can be used by a third round of mutants as a secret handshake. It seems that the whole story might be fairly complex. 
But even if all signals are used and all strategies defect the state, although it is an equilibrium, is not evolutionarily stable. It is a mistake to assume that cheap talk has no effect.

\section{Cheap Talk in a Stag Hunt}

In a note provocatively titled "Nash Equilibria are not Self-Enforcing" , Robert Aumann argues that cheap-talk cannot be effective in the following game:

\begin{tabular}{lll} 
& \multicolumn{2}{l}{ Aumann's Stag Hunt } \\
& $\mathrm{c}$ & $\mathrm{d}$ \\
(Stag) c & 9,9 & 0,8 \\
(Hare) d & 8,0 & 7,7
\end{tabular}

In this base game, there are two pure strategy Nash equilibria, cc and dd. The first is Pareto Dominant and the second is safer (risk-dominant).

Aumann points out that no matter which act a payer intends to do, he has an interest in leading the other player to believe that he will do c. If the other so believes, she will do $\mathrm{c}$ which yields the first player a greater payoff . One can think of $\mathrm{c}$ as hunting stag and $d$ as hunting hare, where diverting the other player to hunting stag increases a hare hunter's chances of getting the hare. Then both stag hunting types and hare hunting types will wish the other player to believe that they are stag hunters.

Aumann concludes that all types of players will send the message, "I am a stag hunter" and consequently that these messages convey no information. In this game, unlike the sender receiver games, we have a principles argument for the ineffectiveness of cheap talk.

The argument, however, is framed in a context different from the evolutionary one we have been considering. Aumann is working within the theory of rational choice, and furthermore is assuming that the signals have a pre-existing meaning, so that the players know which signal says "I am a stag hunter." Does the force of the argument carry over to evolutionary dynamics? We will compare the base game and the resulting cheap-talk game with two signals.

In the base stag hunt game there are three Nash equilibria, both hunt stag, both hunt hare, and a mixed equilibrium. As an evolutionary game we have two evolutionarily stable strategies, hunt stag and hunt hare. The polymorphic state of the population that corresponds to the mixed equilibrium is not evolutionarily stable, and it is dynamically unstable in the replicator dynamics. The dynamical phase portrait is very simple, and is shown in figure 1:

(fig 1 here) 
The state of the system specified by the proportion of the population hunting stag. If $\operatorname{pr}($ Stag $)>7 / 8$ the replicator dynamics carries stag hunting to fixation. If $\operatorname{pr}(\mathrm{stag})<7 / 8$ replicator dynamics carries hare hunting to equilibrium. If $\operatorname{pr}(\mathrm{stag})=7 / 8 \mathrm{we}$ are at an unstable equilibrium of the replicator dynamics.

Now we embed this is a cheap talk game with 2 signals. A strategy now specifies which signal to send, what act to do if signal 1 is received, and what act to do if signal 2 is received. There are 8 strategies in this game. What is the equilibrium structure? First, we must notice that some states where everyone hunts hare are unstable equilibria. For instance, if the entire population has the strategy: "Send signal 1 and hunt hare no matter what signal you receive" then a mutant could invade using the unused signal as a secret handshake. That is, the mutant strategy: "Send signal 2 and hunt stag if you receive signal 2 , but if you receive signal 1 hunt hare" would hunt hare with the natives and hunt stag with its own kind, and would thus do strictly better than the natives. The replicator dynamics would carry the mutants to fixation. Next, neither a population of hare hunters that sends both messages nor a population of stag hunters is at an evolutionarily stable state. The proportions of those who hunt hare and send message 1 to those who hunt hare and send message 2 could change with no payoff penalty. Likewise with the case where all hunt stag. These states are stable is a weaker sense. They are neutrally stable, rather than evolutionarily stable. Under the replicator dynamics we have dynamic stability but not asymptotic stability. None of these states are not attractors (although a set of states, such as the set of states where all hunt stag, can be and is here an attractor).

There is, however, an evolutionarily stable state in the cheap talk game. It is an entirely new equilibrium, which has been created by the signals. This is a state of the population in which half the population has each of the strategies:

$$
\begin{aligned}
& <1 \text {, Hare, Stag }> \\
& <2, \text { Stag, Hare }>
\end{aligned}
$$

The first strategy sends signal 1, hunts hare if it receives signal 1 and hunts stag if it receives signal 2 . The second sends signal 2 , hunts stag if it receives signal 1 and hare if it receives signal 2 . These strategies cooperate with each other, but not with themselves! Notice that in a population that has only these two strategies, the replicator dynamics must drive them to the 50/50 equilibrium. If there are more who play the first strategy, the second gets a greater average payoff; if there are more of the second, the first get a greater average payoff. [This explains why there is no stable equilibrium among the strategies <1,Stag, Hare> and <2,Hare,Stag>, which cooperate with themselves, but not with others. If one were more numerous, it would get a greater payoff, and replicator dynamics would drive it to fixation.]

One can check that this state is evolutionarily stable. Any mutant would do strictly would than the natives and would be driven to extinction by the replicator dynamics. It is also true that this is the only evolutionarily stable state in this game. ${ }^{5}$ We are led to 
wonder whether this is just a curiosity, or whether this new equilibrium plays a significant role in the evolutionary dynamics.

Dynamical questions are also raised by another aspect of Aumann's paper. David Kreps asked Aumann if he would give the same analysis of the ineffectiveness of cheap talk signaling in the following stag hunt game:

\begin{tabular}{lll} 
& \multicolumn{2}{l}{ Kreps' Stag Hunt } \\
& c & d \\
(Stag) c & 100,100 & 0,8 \\
(Hare) d & 8,0 & 7,7
\end{tabular}

Aumann reports: "The question had us stumped for a while. But actually, the answer is straightforward: Indeed, (c,c) is not self-enforcing, even here. It does look better than in figure 1 (Aumann's stag hunt); not because an agreement to play it is self-enforcing, but because it will almost surely be played even without an agreement. An agreement to play it does not improve its chances further. As before, both players would sign the agreement gladly, whether or not they keep it; it therefore conveys no information." Aumann is certainly correct in that in Krep's stag hunt Stag is an attractive choice even without communication. In evolutionary dynamics if most of the population are not initially hunting stag, hare hunting will be carried to equilibrium. The dynamical picture of the base game is shown in figure 2.

(figure 2 here)

But it is also true that in Aumann's stag hunt the odds are rigged for hunting hare. So we might want to also consider a "neutral" stag hunt, where hunting stag and hunting hare are equally attractive:

\begin{tabular}{lll} 
& \multicolumn{2}{l}{ Neutral Stag Hunt } \\
& $\mathrm{c}$ & $\mathrm{d}$ \\
(Stag) c & 15,15 & 0,8 \\
(Hare) d & 8,0 & 7,7
\end{tabular}

The evolutionary equilibrium analysis for Aumann's stag hunt holds good for all these stag hunts. The states in which everyone hunts stag are neutrally stable but not evolutionarily stable. The states in which everyone hunts hare are either unstable (if there is an unused message) or neutrally stable, but they are not evolutionarily stable. The unique evolutionarily stable state is again the polymorphism:

$$
\begin{aligned}
& <1, \text { Hare, Stag }>50 \% \\
& <2 \text {, Stag, Hare }>50 \%
\end{aligned}
$$


It is evident that in this polymorphism, the signals are certainly carrying information. This information allows individuals to always coordinate on an equilibrium of the base game. Ignoring the signals, the frequency of response types [<*, Hare, Stag $>$ or $<*$, Stag , Hare > ] in the population is $50 \%$. The signal sent identifies the response type of the sender with relative frequency of $100 \%$. We should be especially interested to see whether evolutionary dynamics should lead us to expect that this type of state should be rare or frequent.

This leaves us with a number of questions about the size of basins of attraction in the signaling games. Is the size of the basin of attraction of stag hunting behavior (hare hunting behavior) increased, decreased, or unaffected by cheap talk signaling? Does the new polymorphic equilibrium created by signaling have a negligible or a significant basin of attraction?

In a Monte Carlo simulation of 10,000 trials of the neutral stag hunt game, the results were:
All Hunt Stag at Equilibrium
75,386 trials
All Hunt Hare at Equilibrium
13,179 trials
Polymorphism
11,435 trials

It is evident that the basin of attraction of the polymorphic evolutionarily stable state created by the signaling is not negligible. It is almost as great as the basic of attraction of the set of hare-hunting equilibrium states! In the polymorphic equilibrium, the signals carry perfect information about the response type of the sender That information is utilized by the receiver, in that he performs an act that is a best response to the sender's response to the signal he himself has sent.

In an equilibrium in which everyone hunts stag the signals carry no information about response types. Either only one signal is sent, in which case probability of response type conditional on sending that signal is equal to probability of that response type in the population., or both signals are sent, in which case all members of the population have the response type "hunt stag no matter which signal you receive". And in the equilibria where everyone hunts hare both messages are always sent and everyone has the response type "hunt hare no matter which signal you receive".

Nevertheless, the signals have something to do with the basins of attraction of these equilibria. Without signaling stag hunting and hare hunting each have basins of attraction of $50 \%$ of the simplex of possible population proportions. With cheap talk signals the basin of attraction of stag hunting equilibria is above $75 \%$ while the basin of attraction of hare hunting equilibria is below $14 \%{ }^{6}$ There is something of a mystery as to how cheap talk has made this difference. We will pursue this mystery further in the next section. 


\section{Cheap Talk in a Bargaining Game}

We will consider a simple, discrete Nash bargaining game. Each play makes a demand for a fraction of the pie. If their demands total more than 1 , no bargain is struck and they get nothing. Otherwise they get what they demand. In this simplified game there are only three possible demands: $1 / 3,2 / 3$ and $1 / 2$, which we denote by act 1,2 , and 3 respectively. The resulting evolutionary game has a unique evolutionarily stable strategy, Demand $1 / 2$, but it also has an evolutionarily stable polymorphic state in which half of the population demands $1 / 3$ and half of the population demands $2 / 3$. The polymorphic equilibrium squanders resources. Each strategy has an average payoff of $1 / 3$. The state where all demand $1 / 2$ and get it is efficient. Nevertheless, if we compute the basin of attraction in the replicator dynamics of Demand $1 / 2$, it is only about $62 \%$ of the 3 -simplex of possible population proportions. The wasteful polymorphism has a basin of attraction of $38 \%$. (Another polymorphic equilibrium, in which Demand $1 / 3$ has probability $1 / 2$, Demand $1 / 2$ has probability $1 / 6$ and demand $2 / 3$ has probability $1 / 3$, is dynamically unstable and is never seen in simulations.)

What happens if we embed this game in a cheap talk game with 3 signals? A strategy in this cheap talk game is a quadruple:

$<$ Signal to send, Demand if I receive signal 1, Demand if signal 2, Demand if signal 3>

There are now 81 strategies.

If we run as Monte Carlo simulation, sampling from the uniform distribution on the 81-simplex, and let the population evolve for a long enough time, the counterparts of the old 2/3-1/3 polymorphism in the base bargaining games are not seen. Short simulations (20,000 generations) may appear to be headed for such a state, as in example 1 .

\section{Example 1:}

$$
\begin{aligned}
& \mathrm{pr}\langle 2112>=0.165205 \\
& \mathrm{pr}\langle 2122>=0.012758 \\
& \mathrm{pr}\langle 2212>=0.276200 \\
& \mathrm{pr}\langle 2222>=0.235058 \\
& \mathrm{pr}\langle 2312>=0.053592 \\
& \mathrm{pr}\langle 2322\rangle=0.248208
\end{aligned}
$$

All strategies send signal 2 . Approximately half the population demands $1 / 3$ and approximately half demands $2 / 3$. But it is not exactly half, and the numbers do not quite sum to one because very small numbers are not printed out. This simulation $(20,000$ generations) has not run long enough. A population of just these strategies and with half demanding $1 / 3$ and half demanding $2 / 3$ would not be evolutionarily stable because it would be invadible by strategy $\langle 3113\rangle$. Message 3 functions as a "secret handshake". This strategy would get an average payoff of $1 / 3$ when playing against the natives and $1 / 2$ 
when playing against itself. Indeed, when we run the simulation longer the secret handshake strategy has time to grow, and this sort of result is never seen.

As in the previous section, the signals create new evolutionarily stable polymorphic equilibria, such as those in examples 2 and 3:

Example 2:

$$
\begin{aligned}
& \operatorname{pr}\langle 1132\rangle=0.2000000 \\
& \operatorname{pr}\langle 1232>=0.2000000 \\
& \operatorname{pr}\langle 2331>=0.4000000 \\
& \operatorname{pr}\langle 3121>=0.1000000 \\
& \operatorname{pr}\langle 3122\rangle=0.1000000
\end{aligned}
$$

Example 3:

$$
\begin{aligned}
& \text { pr }\langle 1312\rangle=0.250000 \\
& \operatorname{pr}\langle 2213>=0.250000 \\
& \operatorname{pr}\langle 2223>=0.250000 \\
& \operatorname{pr}\langle 3133>=0.250000
\end{aligned}
$$

These appear in simulations where the population was allowed to evolve for 1,000,000 generations.

We can gain some insight into these polymorphisms if we notice that the population can be partitioned into three subpopulations according to signal sent. Since a player conditions her strategy on signal received, a player uses a strategy of the base game against each of these subpopulations, and the choice of strategies against different subpopulations are logically independent.

Consider example 2. The first thing to look at is the interaction of subpopulations with themselves. Considered alone, the subpopulation that sends signal 1 is in the 1/3-2/3 polymorphic evolutionary equilibrium of the base game. So is the subpopulation that sends signal 3. The subpopulation that sends signal 2 is in the All $1 / 2$ evolutionary equilibrium of the base game.

The subpopulations not only play themselves. They also play each other. Notice that when two subpopulations meet they play a pure strategy Nash equilibrium of the base game. When signal 1 senders meet signal 2 senders they both demand 1/2; when signal 1 senders meet signal 3 senders the former demand $2 / 3$ and the latter demand $1 / 3$; when signal 2 senders meet signal 3 senders the former demand $1 / 3$ and the latter demand 2/3. These are all strict equilibria and stable in two population replicator dynamics.

The three subpopulations can be thought of as playing a higher level game with payoff matrix: 


\section{EXAMPLE 2'}

$\begin{array}{llll} & \text { Sig } 1 & \text { Sig2 } & \text { Sig3 } \\ \text { Sig1 } & 1 / 3 & 1 / 2 & 2 / 3 \\ \text { Sig2 } & 1 / 2 & 1 / 2 & 1 / 3 \\ \text { Sig3 } & 1 / 3 & 2 / 3 & 1 / 3\end{array}$

Considered as one-population evolutionary game, this has unique interior attracting equilibrium at $\operatorname{Pr}(\operatorname{Sig} 1)=.4, \operatorname{Pr}(\operatorname{Sig} 2)=.4, \operatorname{Pr}(\operatorname{Sig} 3)=.2$, which are their values in example 2.

Example 3 has a similar analysis. Here the 1/3-2/3 polymorphism occurs in the subpopulation that sends signal 2 , while the other subpopulations always demand $1 / 2$ when they meet themselves. The higher level game between subpopulations has the payoff matrix:

\section{EXAMPLE 3}

$\begin{array}{llll} & \text { Sig } 1 & \text { Sig } 2 & \text { Sig } 3 \\ \text { Sig } 1 & 1 / 2 & 1 / 3 & 2 / 3 \\ \text { Sig } 2 & 2 / 3 & 1 / 3 & 1 / 2 \\ \text { Sig } 2 & 1 / 3 & 1 / 2 & 1 / 2\end{array}$

Considered as a one-population evolutionary evolutionary game, this has a unique interior attracting equilibrium at $\operatorname{Pr}(\operatorname{Sig} 1)=.25, \operatorname{Pr}(\operatorname{Sig} 2)=.50, \operatorname{Pr}(\operatorname{Sig} 3)=.25$, as we observe in example 3.

The foregoing modular analysis depends for its validity on the fact that all the equilibria at various levels of the story are structurally stable dynamical attractors.

These polymorphisms achieve a payoff much better than that of the $1 / 3-2 / 3$ polymorphism in the base game. In the $1 / 3-2 / 3$ polymorphism, each strategy has an average payoff of $1 / 3$. In examples 2 and 3 , the average payoffs to a strategy are $0.466666 \ldots$ and $0.458333 \ldots$ respectively. The remaining inefficiencies are entirely due to the polymorphisms involved in subpopulations of those who send a specified signal meeting themselves.

Although these new polymorphisms are fascinating, they play a minor role in the overall evolutionary dynamics of the bargaining game. In fact, more that $98 \%$ of the 81 simplex of the cheap talk game evolves to one of the equilibria where all demand 1/2. The result is robust to the deletion or addition of a signal. If we run the dynamics with 2 signals or 4 signals, the resulting simulation still leads to all demanding $1 / 2$ more than $98 \%$ of the time. The little mystery that we were left with at the end of the last section has become a bigger mystery. 
Existing theorems about cheap-talk do not apply. ${ }^{7}$ The simulation is set up so that each run begins in the interior of the 81-simplex. That is to say that each strategy has some positive, possibly small, probability. In particular, there are no unused messages. The game is not a game of common interest. It is in the common interest of the players to make compatible demands, but within these bounds there is opposition of interests. The best result is to demand $2 / 3$ and while the other player demands $1 / 3$.

Signals have no preexisting meaning. Does meaning evolve? Consider some of the final states of trials in which the population ends up in states where everyone demands $1 / 2$ : Example 4 (1,000,000 generations):

$$
\begin{aligned}
& \operatorname{pr}\langle 2131>=0.189991 \\
& \operatorname{pr}\langle 2132>=0.015224 \\
& \operatorname{pr}\langle 2133>=0.037131 \\
& \operatorname{pr}\langle 2231>=0.245191 \\
& \operatorname{pr}\langle 2232>=0.048024 \\
& \operatorname{pr}\langle 2233>=0.021732 \\
& \operatorname{pr}\langle 2331>=0.128748 \\
& \operatorname{pr}\langle 2332>=0.175341 \\
& \operatorname{pr}\langle 2333>=0.138617
\end{aligned}
$$

Each strategy sends signal 2; each strategy demands half upon receipt of signal 2. But concerning what would be done upon receipt of the unused signals 1 and 3, all possibilities are represented in the population.

Example $5(1,000,000)$ generations):

$$
\begin{aligned}
& \text { pr }\langle 1333>=0.770017 \\
& \text { pr }\langle 2333>=0.057976 \\
& \operatorname{pr}\langle 3333>=0.172008
\end{aligned}
$$

Here all messages are sent, any each strategy ignores message sent and simply demands half. In between these two extremes we find all the sorts of intermediate cases.

It is clear that in our setting we do not have anything like the spontaneous generation of meaning that we see in Lewis sender-receiver games. The action of signals here is more subtle. Suppose we shift our attention from the strong notion of meaning that we get from a signaling system equilibrium in a sender-receiver game to the weaker notion of information. A signal carries information about a player if the probability that the player is in a certain category given the he sent the signal is different from the probability that he is in the category simply given that he is in the population. At equilibria where players all demand half, signals cannot carry information about the player's acts. Conceivably, signals could carry some information about a player's response type at equilibrium, where response type consists of the last three coordinates of a player's 
strategy. But in examples 4 and 5 this cannot be true, because in example 4 there is only one signal sent and in example 5 there is only one response type.

Perhaps the place to look for information in the signals is not at the end of the process, but at the beginning and middle of evolution. For the signals to carry no information about response types at a randomly chosen vector of population proportions would take a kind of a miracle. At almost all states in the simplex of population proportions there is some information about response types in the signals. There is information present - so to speak, by accident - at the beginning of evolution in our simulations. Any information about a response type could be exploited by the right kind of strategy. And the right kind of strategy is present in the population because all types are present. Strategies that exploit information present in signals will grow faster than other types. Thus, there will be an interplay between "accidental" information and the replicator dynamics.

To investigate this idea we need a quantification of the average amount on information in a signal. We use the Kullbach-Leibler (K-L) discrimination information between the probability measure generated by the population proportions and that generated by conditioning on the signal.

Let $\mathrm{P}$ denote the proportion of the population and $\mathrm{p}_{\mathrm{i}}$ denote the proportion of the sub-population that sends message i. Recall that a response type consists of a triple:

$<$ Act if message i, Act if Message 2, Act if Message 3>

There are 3 signals which we denote as $S_{i}$ and 27 response types, which we denote as $T_{k}$. Then the K-L information in message $\mathrm{i}$ is:

$$
{ }_{k}\left(\mathrm{p}_{\mathrm{i}}\left[\mathrm{T}_{\mathrm{k}}\right] \log \left(\mathrm{p}_{\mathrm{i}}\left[\mathrm{T}_{\mathrm{k}}\right] / \mathrm{P}\left[\mathrm{T}_{\mathrm{k}}\right]\right)\right.
$$

The average amount of information in the signals in the population is gotten by averaging over signals: ${ }^{8}$

\section{i $P\left[S_{i}\right] \quad k\left(p_{i}\left[T_{k}\right] \log \left(p_{i}\left[T_{k}\right] / P\left[T_{k}\right]\right)\right.$}

This is identical to the information provided by an experiment, as defined by Lindley (1956), where looking at a signal is thought of an as experiment and the particular signal seen is the experimental outcome.

For example, consider the polymorphic equilibrium in the stag hunt game:

$$
\begin{aligned}
& <1, \text { Hare, Stag }>50 \% \\
& <2, \text { Stag, Hare }>50 \%
\end{aligned}
$$

There are two response types present in the population and two signals. In the probabilities conditional on signal 1 , there is only one response type. The information in signal 1 is just: 


$$
1 \log (1 /(1 / 2))=\log (2)=.693 \quad \text { (natural logarithm) }
$$

The information in signal 2 is likewise $\log (2)$ as in the average information in the signals in the population. In example 4 of this section, the average information in the population is the information in signal 2, which is always sent. This is zero, since for all response types, $\mathrm{k}$, in the population $\mathrm{p}_{\mathrm{k}}=\mathrm{P}$. In example 5 of this section there is only one response type in the population, so the information in each message is zero. It is, however, possible for there to be positive information in an equilibrium population state in which everyone demands $1 / 2$, for instance:

$$
\begin{array}{ll}
<1,3,3,1> & 50 \% \\
<2,3,3,2> & 50 \%
\end{array}
$$

Only messages 1 and 2 are sent, and the response types only differ on what they would do counterfactually, in response to receiving message 3 . The messages distinguish these response types perfectly, so they contain information about response types.

It is possible to compute the average information in the messages in a population, and to average the results over many trials in the Monte Carlo simulation used to compute basins of attraction. The results of averaging over 1000 trials are shown in figure 3. (The computation uses the natural logarithm.)

\section{(figure 3 "Evolution of Information" here)}

There is, of course, some information present at the onset of the trials "by accident". Then the replicator dynamics leads to an increase in average information in the signals which peaks at about 300 generations. After that the average information in a signal begins a slow, steady decline.

What is the effect of this information? If there is information present in the population there are strategies present that can use it to good effect. Thus, if we look only at the behaviors in the base bargaining game -as if cheap talk was invisible -we would expect to see some departure from random pairing. That is to say, the signals should induce some correlation in the behaviors in the bargaining game. We might look to see whether Demand $1 / 2$ behaviors are exhibited in pairs more often than at random, which would favor those types that manage to effect that the correlation. We also might see if there is positive correlation between demand 2/3 and demand 1/3 behaviors. And we should be interested in whether negative correlation develops between $1 / 2$ and $2 / 3$ players and between pairs of $2 / 3$ players. There would be something to be said for all the foregoing if the behaviors were fixed and self-interested. But they are not, and the evolution of behaviors is a complex product of the evolution of signal and response. How the dynamics of all this will work out is difficult to see a priori. So we again resort to simulation. Here we calculate the covariance of the indicator variables for behaviors.

That is to say, two individual types from the 81 types in the signaling game are picked according to the current population proportions. A pair of types determines the 
behaviors in the bargaining game. There are indicator variables for "First member demands $2 / 3,1 / 3,1 / 2$ ", and for "Second member demands $2 / 3,1 / 3,1 / 2$ ". Then:

$$
\operatorname{COV}(\mathrm{i}, \mathrm{j})=\operatorname{Pr}(\mathrm{i}, \mathrm{j})-\operatorname{Pr}(\mathrm{i})(\operatorname{Pr}(\mathrm{j})
$$

where $\operatorname{Pr}(\mathrm{i}, \mathrm{j})$ is the probability that the first demands $\mathrm{i}$ and the second demands $\mathrm{j}$. [note: $\operatorname{Pr}(\mathrm{i}, \mathrm{j})=\operatorname{Pr}(\mathrm{j}, \mathrm{i})$ since the draws of types are independent so $\operatorname{Cov}(\mathrm{i}, \mathrm{j})=\operatorname{Cov}(\mathrm{j}, \mathrm{i})$

Demand $1 / 2$ behaviors would "like" to be paired with themselves; Demand $2 / 3$ behaviors would "like" to be paired with Demand 1/3 behaviors; Demand $1 / 2$ behaviors and Demand $2 / 3$ behaviors would "like" to avoid one another. Figure 4 shows that evolution complies to some extent. (It shows the results of simulations averaged over 1000 trials.)

\section{(figure 4 "Evolution of Correlation I" here)}

The interaction between the information in the signals and the evolutionary dynamics generates a positive correlation between the compatible demands $(1 / 2,1 / 2)$ and $(2 / 3,1 / 3)$. It generates a negative correlation between the incompatible demands $(1 / 2,2 / 3)$. In each of these cases, the absolute magnitude of the covariance peaks at about 400 generations. At its peak, $\operatorname{Cov}(1 / 2,1 / 2)$ is above $3 \%$ while $\operatorname{Cov}(2 / 3,1 / 3)$ is less than half of that value.

One should not be hasty, however, in generalizing the compliance of the correlation generated by evolution with what one might expect a strategist to desire. Demand $1 / 2$ behaviors would not at all mind meeting compatible Demand $1 / 3$ behaviors. And Demand 2/3 behaviors would "like" to avoid meeting themselves. But here evolution fails to deliver correlations in the desired directions - as shown in figure 5:

\section{(figure 5:"Evolution of Correlation II" here)}

$\operatorname{Cov}(1 / 2,1 / 3)$ is a negative at 400 generations as $\operatorname{Cov}(2 / 3,13)$ is positive. And evolution does not give Demand $2 / 3$ behavior the edge in avoiding itself that one might expect. Demand 2/3 behaviors do not effectively use signals to avoid each other. $\operatorname{Cov}(2 / 3,2 / 3)$ is mostly positive but near zero. It is evident from figure 5 that thinking about what behaviors in the embedded game would "like to happen" is not a reliable guide to the working of the evolutionary dynamics in the cheap talk game.

The evolution of correlation can be put into perspective by comparing it to the evolution of bargaining behaviors, with the probabilities averaged over the same 1000 trials. This is shown in figure 6.

$$
\text { (figure } 6 \text { "Evolution of Bargaining Behaviors" here) }
$$

Demand $1 / 2$ behaviors rapidly take over the population. Demand $2 / 3$ behaviors die out even more quickly that demand $1 / 3$ behaviors, notwithstanding the positive correlation between the two. 
At 400 generations Demand $1 / 2$ has, on average, taken over $73 \%$ of the population; at 1000 generations it has, on average $97 \%$ of the population. At 2000 generations, positive and negative covariance have all but vanished, Demand $1 / 2$ behaviors have taken over $99 \%$ of the population, and what little information remains in the signals is of little moment. In the limit, the information left in the signals, if any, is counterfactual. It only discriminates between response types that differ in their responses to signals that are not sent.

It is clear that the interesting action is taking place at about 400 generations. This suggests that we might look at individual orbits at 400 generations and then at (or close to) equilibrium. Here is an example to show the complexity of the interaction of signaling strategies that set of correlations. It is of a printout of all strategies with more than $1 \%$ of the population at 400 generations and at 10,000 generations of a single run, followed by their population proportion and average payoff At 400 generations, we have:

$<1311>0.011134 \mathrm{U}=0.386543$

$<1313>0.044419 \mathrm{U}=0.436378$

$<1323>0.012546 \mathrm{U}=0.398289$

$<1333>0.203603 \mathrm{U}=0.473749$

$<2133>0.026870 \mathrm{U}=0.372952$

$<2311>0.52620 \quad \mathrm{U}=0.385357$

$<2312>0.014853 \mathrm{U}=0.369061$

$<2313>0.025405 \mathrm{U}=0.425317$

$<2323>0.054065 \mathrm{U}=0.416539$

$<3113>0.022481 \quad \mathrm{U}=0.354270$

$<3311>0.016618 \mathrm{U}=0.3954966$

$<3312>0.046882 \mathrm{U}=0.396710$

$<3313>0.050360 \mathrm{U}=0.416904$

$<3322>0.010570 \mathrm{U}=0.366388$

$<3 \quad 323>0.011266 \mathrm{U}=0.386582$

$<3331>0.124853 \mathrm{U}=0.419688$

$<3333>0.010625 \mathrm{U}=0.440625$

At 10,000 generations, we have:

$<1333>0.973050 \mathrm{U}=0.500000$

(with the remaining population proportions spread other the other 80 strategies.)

It is evident that there is more going on here than the secret handshake. Handshake strategies are present. For example, the first strategy listed, < 1311 1 sends signal one, demands half when paired with anyone who sends signal one, and plays it safe by demanding $1 / 3$ in all other cases. But there are also what might be though of as antihandshake strategies, such as $<3331>$, which occupies about $12 \frac{1}{2} \%$ of the population at 400 generations. This strategy demands $1 / 2$ when it meets those who send a different signal, but plays it safe by only demanding $1 / 3$ when it meets those who send the same signal. The anti-handshake strategy contributes to the success of the strategy that 
eventually takes over almost all of the population $\left\langle\begin{array}{lllll} & 3 & 3 & 3\end{array}\right\rangle$ as it eventually dies out. The same is true of the handshake strategy $<\begin{array}{llll}1 & 3 & 1 & 1>\end{array}{ }^{9}$

What is the cause of the dramatic difference in the magnitude basins of attraction of the equal split produced by cheap talk (between 62\% AND 98+\%)? We would like to have a more complete analysis, but we can say something. It appears to be due to transient information. By way of a complex interaction of signaling strategies, this transient information produces transient covariation between behaviors in the base bargaining game. Much more than the "secret handshake" is involved. The type of covariation produced could not have been a priori on grounds of rational choice. Nevertheless, the net effect is to increment the fitness of strategies that demand $1 / 2$.

\section{Conclusion}

Cheaptalk matters! Costless signals can have a large effect on evolutionary dynamics, even in situations where rational choice theory predicts that they should be ineffective. The signals may create entirely new equilibria, and may change the stability properties of equilibria in the base game. Equilibrium analysis, however, misses important dynamic effects of signaling. Costless signals may cause large changes the relative magnitude of basins of attraction of multiple equilibria in the base game.

In the evolutionary process information that is present "by accident" is used and amplified in the evolutionary process. Sometimes informative signaling goes to fixation. This is the case in polymorphic equilibria whose existence is created by the signals. But sometimes the information is transient, and has disappeared by the time the dynamics has reached a rest state.

Transient information matters! Information, although transient, may nevertheless be important in determining the eventual outcome of the evolutionary process. Why and how this is so is a question involving complex interaction of signaling strategies. Some insight into the nature of these interactions can be gained from simulations, but a deeper analysis would be desirable.

We can say this for certain. If costless pre-play signaling is present, then omitting it from the model on rational choice grounds may be a great error. If the signals were invisible, and an observer only saw the behaviors in the base games we have discussed, the course of evolution would not be intelligible. Mysterious correlations would come and go. The dynamics would not appear to be the replicator dynamics. Signals, even when they are cheap, have a crucial role to play in evolutionary theory. 


\section{References}

Alexander, J. and Skyrms, B. (1999) "Bargaining with Neighbors: Is Justice Contagious?" Journal of Philosophy 588-598.

Aumann, R. J. (1990) "Nash Equilibria are Not Self-Enforcing" in Economic Decision Making, Games, Econometrics and Optimization. ed. J.J.Gabzewicz, J.-F. Richard and L.A.Wolsey North Holland:Amsterdam 201-206.

Bhaskar, V. (1998) "Noisy Communication and the Evolution of Cooperation" Journal of Economic Theory 82, 110-131.

Banerjee, A, and Weibull, J. (2000) "Neutrally Stable Outcomes in Cheap-Talk Coordination Games" Games and Economic Behavior 32, 1-24.

Blume, A., Kim, Y-G., and Sobel, J. (1993) "Evolutionary Stability in Games of Communication" Games and Economic Behavior 5, 547-575.

Crawford, V. and Sobel, J. (1982) "Strategic Information Transmission" Econometrica 50, 1431-1451.

Grafen, A. (1990) "Biological Signals as Handicaps" Journal of Theoretical Biology 144: 517-546.

Kim, Y-G. and Sobel, J. (1995) "An Evolutionary Approach to Pre-play Communication" Econometrica 63, 1181-1193.

Kullback, S. (1959) Information Theory and Statistics Wiley: New York.

Kullback, S. and Leibler, R. A. (1951) "On Information and Sufficiency” Annals of Mathematical Statistics 22, 79-86.

Lewis, D. K. (1969) Convention: A Philosophical Study. Oxford: Blackwell.

Lindley, D. (1956) "On A Measure of the information Provided by an Experiment" Annals of Mathematical Statistics 27, 986-1005.

Nydegger, R. v. and Owen, G. (1974) "Two-Person Bargaining: An Experimental Test of the Nash Axioms" International Journal of Game Theory III 239-250.

Robson, A. J. (1990) "Efficiency in Evolutionary Games: Darwin, Nash and the Secret Handshake" Journal of Theoretical Biology 144, 379-396.

Roth, A. and Malouf, M. (1979) "Game Theoretic Models and the Role of Information in Bargaining" Psychological Review LXXXVI 574-94. 
Schlag, K. (1993) "Cheap Talk and Evolutionary Dynamics" Discussion Paper. Bonn University.

Schlag, K. (1994) "When Does Evolution Lead to Efficiency in Communication Games?" Discusssion Paper. Bonn University.

Skyrms, B. (1996) Evolution of the Social Contract. N.Y.: Cambridge.

Skyrms, B. (1999) "Stability and Explanatory Significance of Some Simple Evolutionary Models" Philosophy of Science 67, 94-113.

Sobel, J. (1993) "Evolutionary Stability and Efficiency" Economic Letters 42, 301-312.

Taylor, P. and Jonker, L. (1978) "Evolutionarily Stable Strategies and Game Dynamics" Mathematical Biosciences 40, 145-156.

Van Huyck, J., Batallio, R. Mathur, S., Van Huyck, P. and Ortmann, A. (1995) "On the Origin of Convention: Evidence from Symmetric Bargaining Games" International Journal of Game Theory XXXIV 187-212.

Warneryd, K. (1991) "Evolutionary Stability in Unanimity Games with Cheap Talk" Economic Letters 39, 295-300.

Warneryd, K. (1993) "Cheap Talk, Coordination and Evolutionary Stability" Games and Economic Behavior 5, 532-546.

Zahavi, A. (1975) "Mate selection - a Selection for a Handicap" Journal of Theoretical Biology 53, 205-214.

Zahavi, A. and Zahavi, A. (1997) The Handicap Principle. Oxford: Oxford University Press.

Notes: 
${ }^{1}$ Zahavi (1975), Grafen (1990), Zahavi and Zahavi (1997).

${ }^{2}$ A more general sender-receiver was introduced and analyzed in Crawford and Sobel (1982).

${ }^{3}$ The situation is somewhat complicated if the Lewis model is modified to allow the number of messages to exceed the number of states, but the analysis by Wärneryd shows how the privileged status of signaling system equilibria remains intact.

${ }^{4}$ Skyrms (1999).

${ }^{5}$ See Schlag (1993) and Banerjee and Weibull (2000).

${ }^{6}$ In Aumann's stag hunt the basin of attraction of stag hunting without cheap talk is only .125. With cheap talk the basin of attraction of stag hunting is increased to .149. The polymorphic Evolutionarily Stable State is also seen here, with a basin of attraction of .015 .

${ }^{7}$ Bjaskar (1998), Blume, Kim and Sobel (1993), Kim and Sobel (1995), Schlag (1993), (1994), Sobel(1993), Warneryd (1991)(1993).

${ }^{8}$ The sums are over those signals that are present in some positive proportion of the population.

${ }^{9}$ The reader may wonder, as I did, what the effect would be of removing all potential handshake strategies. I ran simulations where, in the first generation, I killed every strategy that demanded $1 / 2$ when it met itself. Nevertheless, about $38 \%$ of the time the population converged to an equilibrium where each strategy demanded $1 / 2$ of every other strategy that sent a different signal. For each signal, there were two types equally present, which implement the $1 / 2-2 / 3$ polymorphism within the sub-population that sends that signal. Here is an instance:

$\langle 1133\rangle \quad \mathrm{pr}=.01667$

$\langle 1233\rangle \quad \mathrm{pr}=.01667$

$\langle 2313\rangle \quad \mathrm{pr}=.01667$

$\langle 2323\rangle \quad \mathrm{pr}=.01667$

$<3331\rangle \quad$ pr $=.01667$

$<3332>\quad$ pr $=.01667$

The average payoff for each strategy is .444444 . 


\section{Aumann's Stag Hunt}

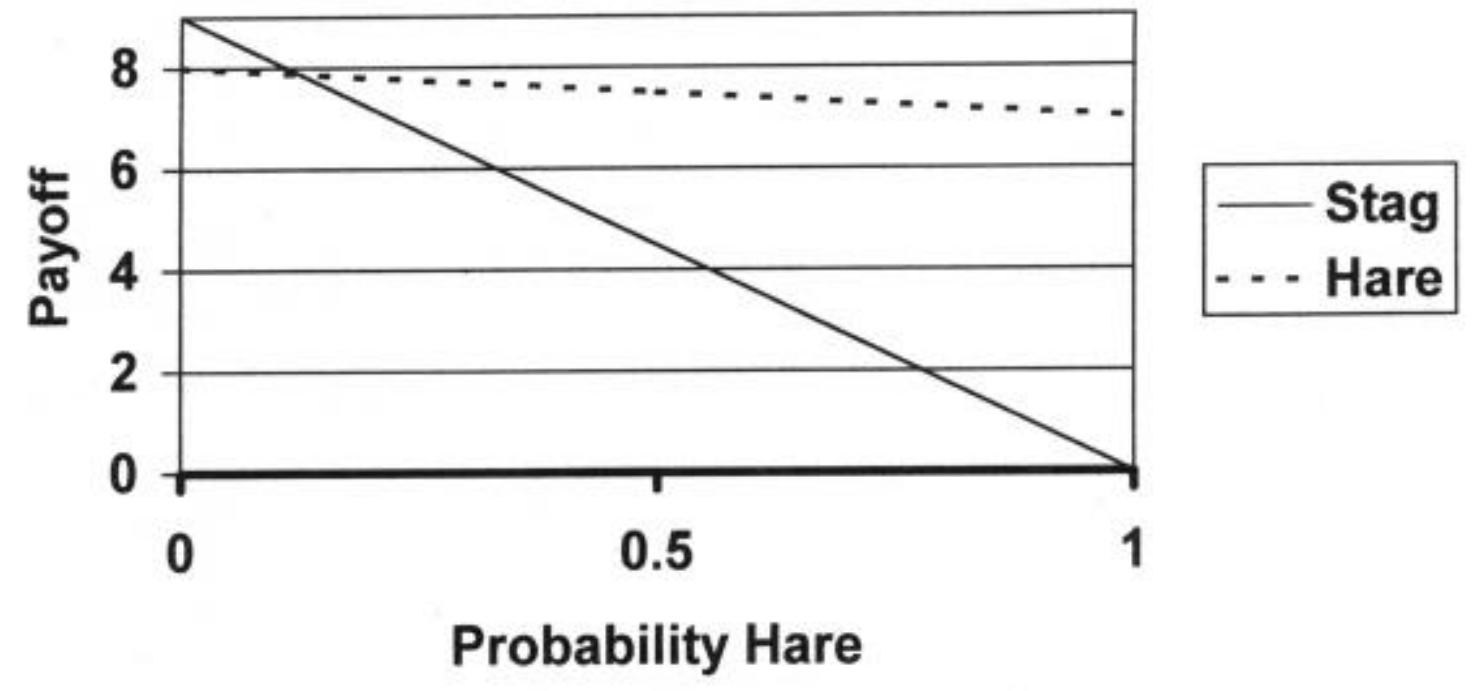

figure 1 


\section{Kreps' Stag Hunt}

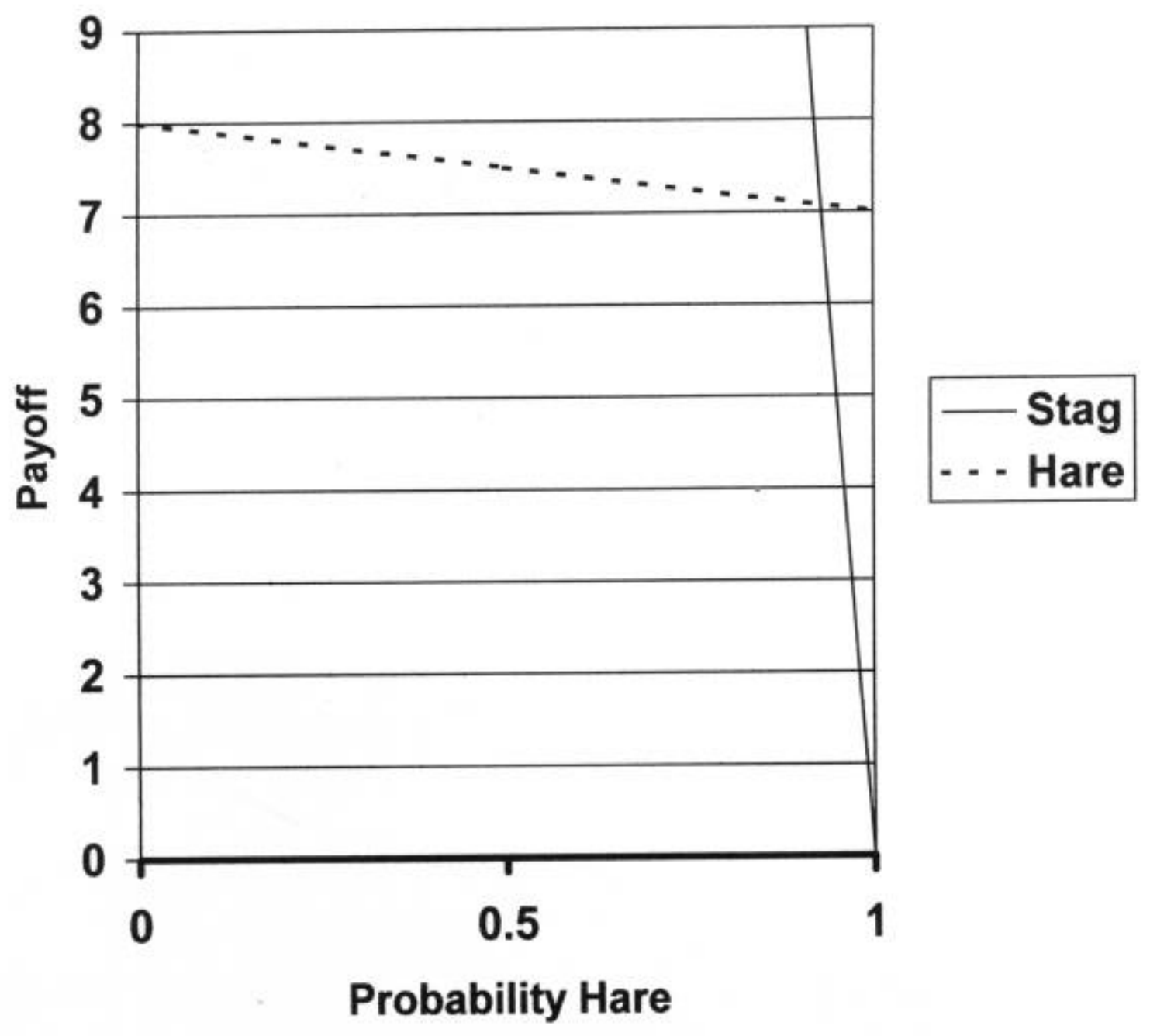

figure 2 
Evolution of Information

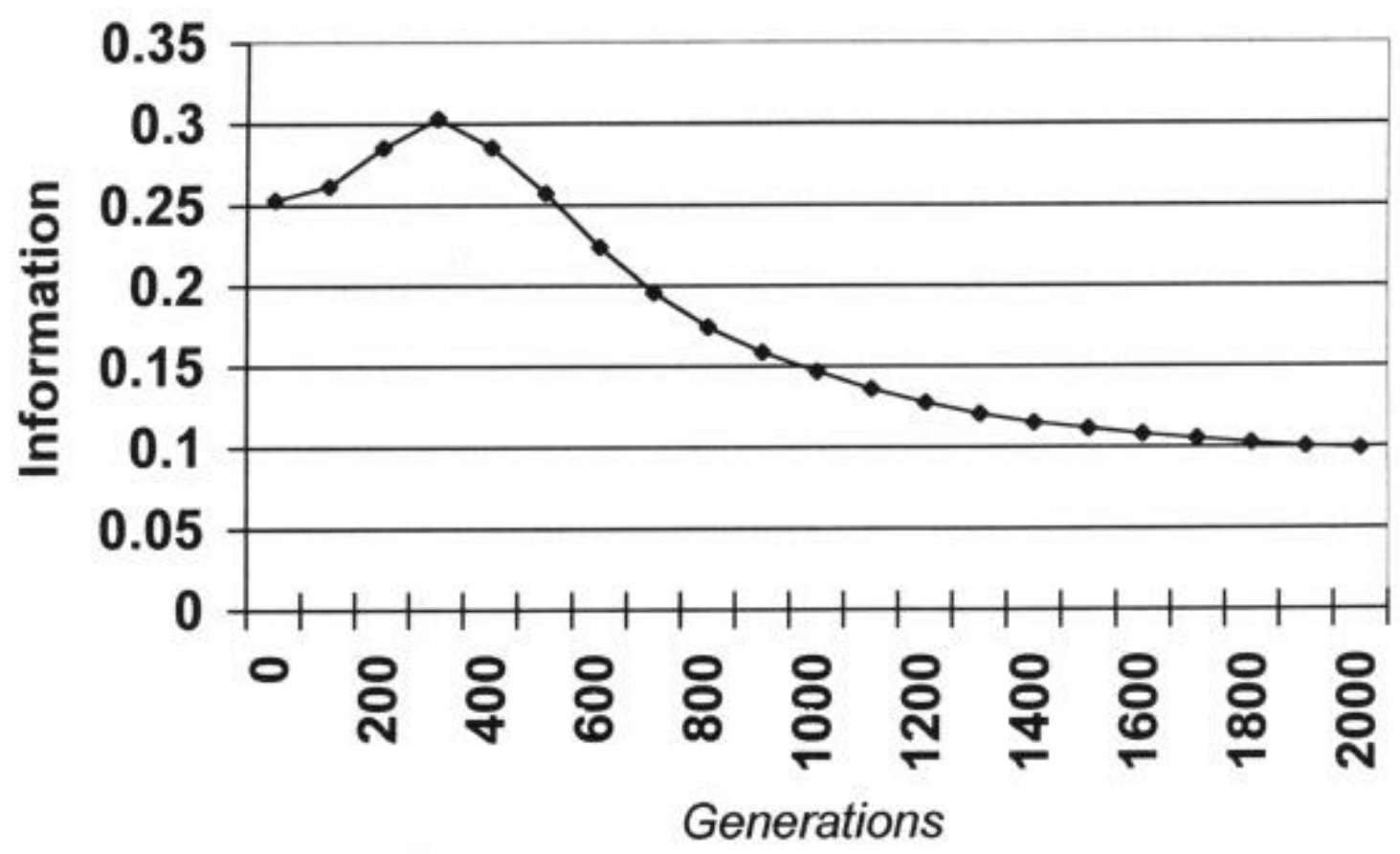

figure 3 


\section{Evolution of Correlation I}

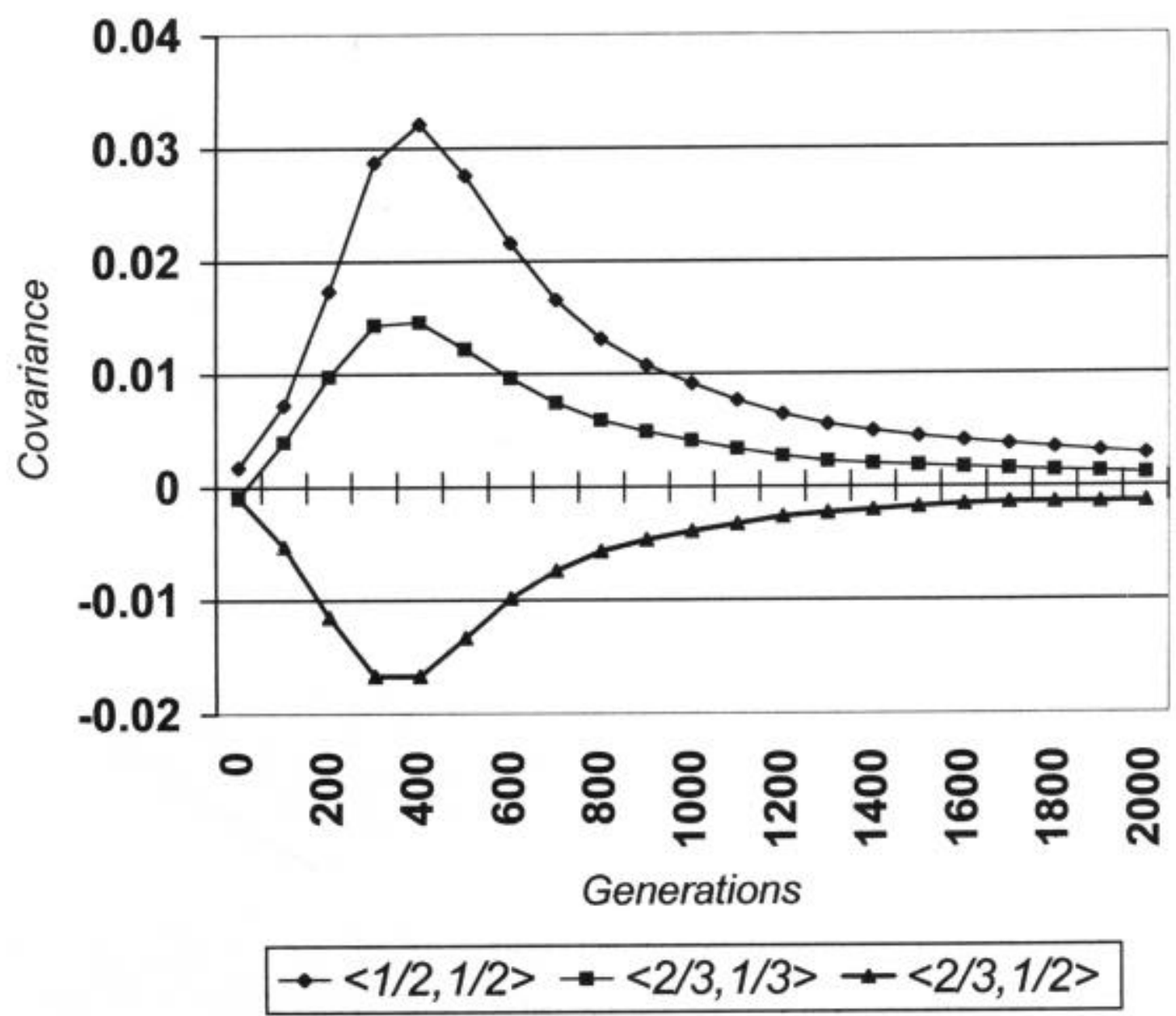

figure 4 


\section{Evolution of Correlation II}

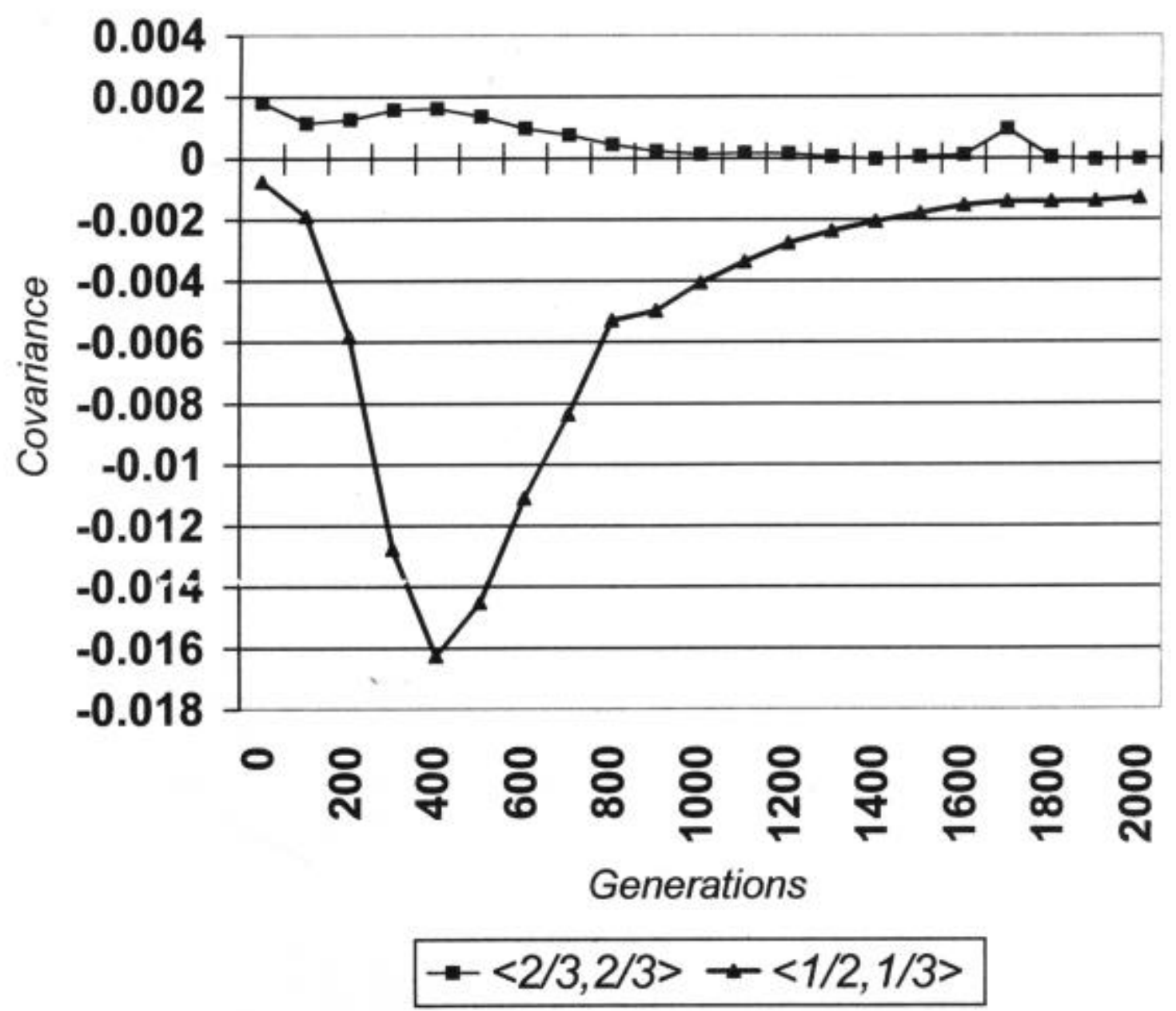

figure 5 


\section{Evolution of Bargaining Behaviors}

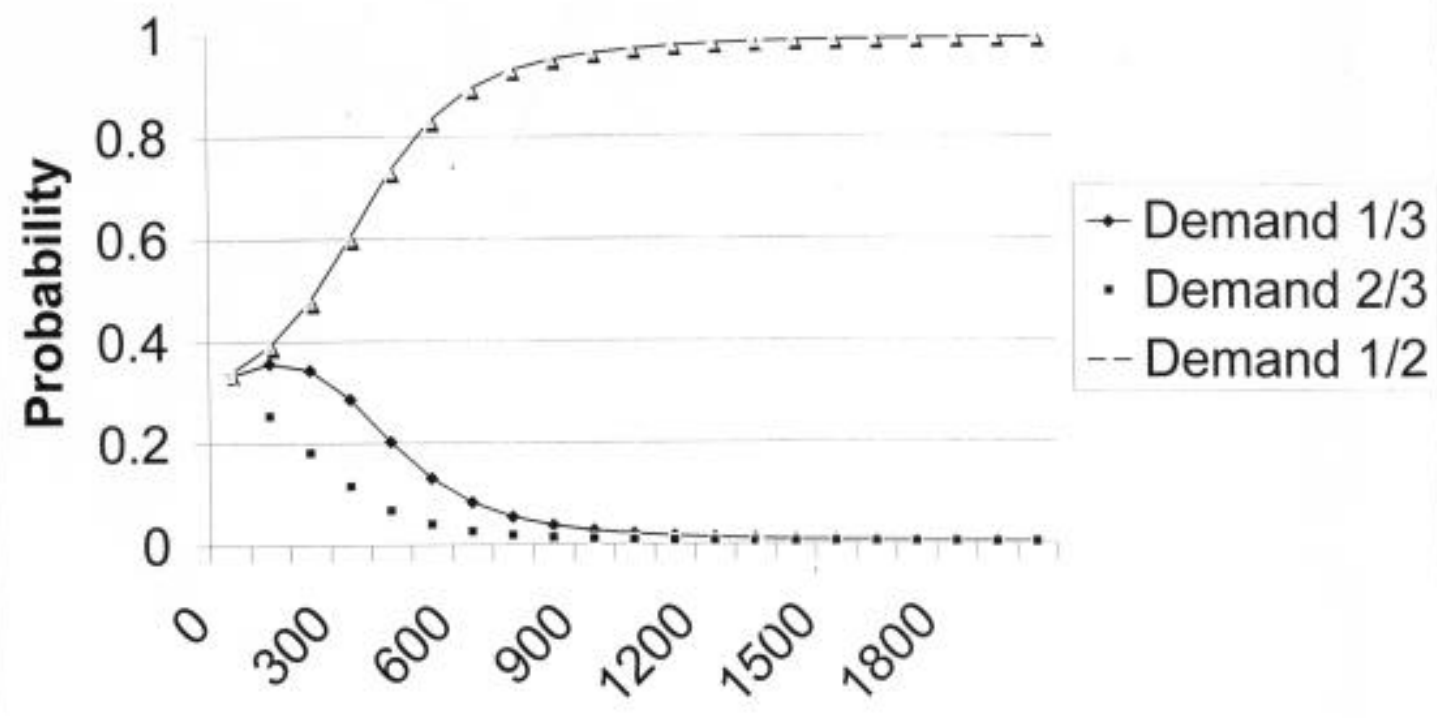

Generation

figure 6 